\title{
Spinal cellular and network properties modulate pain perception
}

\author{
Pascal Darbon ${ }^{a}$ \\ Institut des Neurosciences Cellulaires et Intégratives, CNRS - Université de Strasbourg, France
}

\section{Abstract}

Through the example the modulation of pain perception by a steroid hormone, I'm going to show you a multiscale interaction between two systems of an organism. Indeed, we will see how a the endocrine system via a molecule circulating in the blood will modulate its target molecule in the nervous system that will, in turn, change cellular activities at one stage of the central nervous system that could alter the behavior of this organism.

Indeed our body collects somatosensory information from our environment that will be transmitted to the central nervous system by the peripheral nervous system. Amongst these sensory information there are nociceptive sensory inputs. A nociceptive stimuli detected at the level of the skin will be relayed by the spinal cord to the cortex where it will be interpreted as pain. The dorsal horn of the spinal cord is not a mere relay of the primary nociceptive input. At the level of the superficial layers of the dorsal horn, the nociceptive inputs are processed by a network of excitatory and inhibitory neurons before being transmitted via an ascending pathway to the brain where it will be interpreted as pain. At that level nociceptive processing is tuned by GABAA receptor-mediated inhibition in the spinal cord dorsal horn. These GABAergic inhibitory postsynaptic currents (IPSCs) are known to be modulated by spinally reduced steroids.

Corticosterone (CORT) is a glucocorticoid produced by adrenal glands under the control of the hypothalamic-pituitary-adrenal axis. Circulating CORT can enter the central nervous system and be reduced to neuroactive reduced steroids, which modulate GABAA receptors. In the dorsal spinal cord, GABAergic transmission modulates integration of nociceptive information. It has been shown that enhancing spinal inhibitory transmission alleviates hyperalgesia and allodynia. Therefore, the spinal neuronal network is a pivotal target to counteract pain symptoms. Thus, any increase of spinal reduced steroids production enhancing GABAergic inhibition should reduce nociceptive messages integration and pain response.

Previously, it has been shown that high levels of plasma glucocorticoids give rise to analgesia. However to our knowledge nothing has been reported regarding a direct non genomic modulation of neuronal spinal activity by peripheral CORT. In the present study, we used combined in vivo and in vitro electrophysiology approaches, associated with the measure of nociceptive mechanical sensitivity and plasma corticosterone level measurement to assess the impact of circulating CORT on rat nociception. We showed that CORT plasma level elevation produced analgesia via the reduction of nociceptive fiber mediated spinal responses. CORT is spinally reduced in the neuroactive metabolite THDOC that specifically enhances lamina II GABAergic synaptic transmission. The main

\footnotetext{
${ }^{\text {a }}$ Corresponding author: pdarbon@inci-cnrs.unistra.fr
} 
consequence is a reduction of lamina II network excitability reflecting a selective decrease in processing of nociceptive inputs. The depressed neuronal activity at the spinal level then in turn leads to a weaker nociceptive message transmission to supraspinal structures and hence to an alleviation of pain. 